\title{
DESIGN AND CHARACTERIZATION OF A NOVEL KNEE ARTICULATION MECHANISM
}

\author{
M. OLINSKI*, A. GRONOWICZ and A. HANDKE \\ Wrocław University of Science and Technology \\ Faculty of Mechanical Engineering \\ Department of Biomedical Engineering \\ Mechatronics and Theory of Mechanisms \\ Lukasiewicza St. 7/9, 50-371 Wrocław. POLAND
}

E-mails: michal.olinski@pwr.edu.pl, antoni.gronowicz@pwr.edu.pl,artur.handke@pwr.edu.pl

\author{
M. CECCARELLI \\ University of Cassino and South Latium \\ Department of Civil Engineering and Mechanics \\ LARM: Laboratory of Robotics and Mechatronics \\ Via G Di Biasio 43, Cassino (FR). ITALY \\ E-mail: ceccarelli@unicas.it
}

\begin{abstract}
The paper is focused on designing a novel controllable and adjustable mechanism for reproducing human knee joint's complex motion by taking into account the flexion/extension movement in the sagittal plane, in combination with roll and slide. Main requirements for a knee rehabilitation supporting device are specified by researching the knee's anatomy and already existing mechanisms. A three degree of freedom (3 DOF) system (four-bar like linkage with controlled variable lengths of rockers) is synthesised to perform the reference path of instantaneous centre of rotation (ICR). Finally, a preliminary design of the adaptive mechanism is elaborated and a numerical model is built in Adams. Numerical results are derived from simulations that are presented to evaluate the accuracy of the reproduced movement and the mechanism's capabilities.
\end{abstract}

Key words: knee joint, 3 DOF linkage, synthesis, optimization, numerical experiments.

\section{Introduction}

The human knee is the largest and one of the most complicated articulations in the human body. The movement of the knee joint involves abduction/adduction, as well as internal/external rotation, but it can be simplified to 1 DOF (one degree of freedom) with the main movement of flexion/extension in the body's sagittal plane. It has already been discovered that even this movement is not just a simple rotation in a hinge. In fact, it constitutes a more complex combination of slide and roll, emerging from the knee's anatomy, particularly from the shape of cooperating joint surfaces of the femur and tibia bones and the cruciate ligaments (Fig.1). For this reason the instantaneous centre of rotation (ICR) of the knee joint changes its position with the angle of flexion. The trajectory of ICR is different for each person and can also vary for one person depending on various conditions, like for instance the loading of the leg with outside forces.

In the past the knee's complex motion was not always seriously taken into consideration, for instance in designing the devices supporting the knee joint like orthoses or assisting in exercises and movement based rehabilitation (kinesiotherapy). Since then, much research has been carried out on understanding this movement and applying it into devices. The knee's trajectory during walking can be characterized with various measurement systems, for example CaTraSys (Varela et al. [1]). Nowadays, this knowledge is

\footnotetext{
* To whom correspondence should be addressed
} 
common and orthotic devices use various mechanisms to reproduce the approximately proper motion. The well-known knee model based on a crossed four-bar mechanism is partially derived from the knee's anatomy, particularly the anterior and posterior cruciate ligaments behaviour may be simplified by two crossed bars (Fig.2) (Ciszkiewicz and Knapczyk [2]).

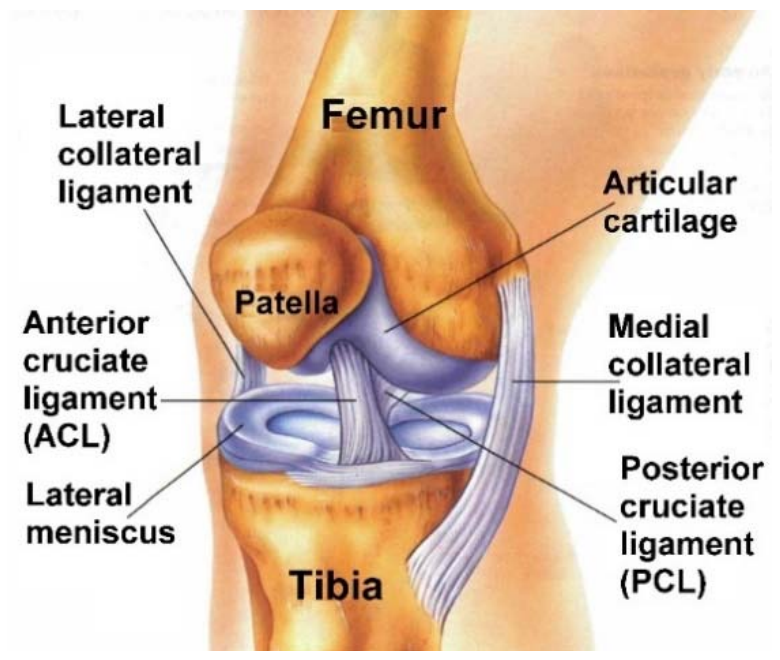

Fig.1. The human knee joint's anatomy with visible cruciate ligaments.

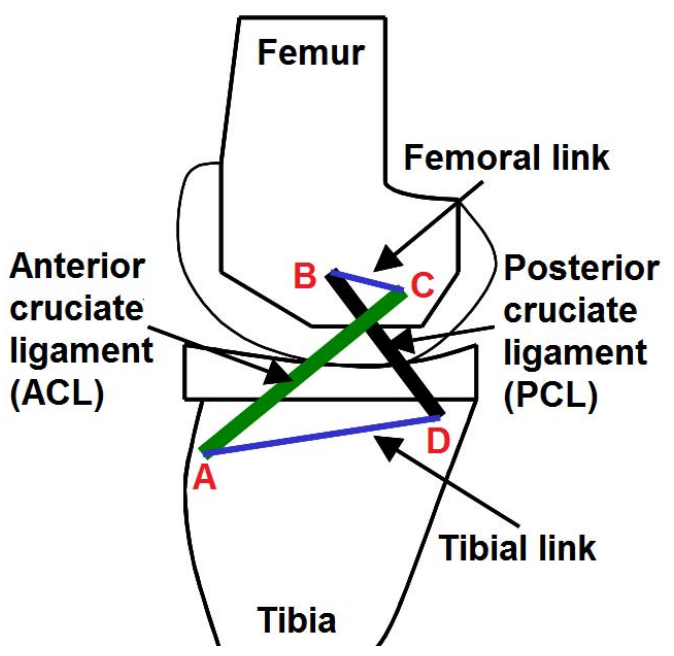

Fig.2. The left knee with an equivalent crossed four-bar mechanism.

In the case of exoskeletons that are used by healthy people for walking or increasing the strength and endurance, such an accurate reproduction of the joint's movement is not necessary and for most of designed devices it is not taken into account (Lovasz et al. [3]; Liang et al. [4]). As regards the kinesiotherapy and biomechanics, the knee joint is also often simplified and considered as a hinge (Tate [5]). However, in these cases it is not considered a proper model, since unnatural movement may be exerted on the knee causing discomfort and pain. It may even damage the internal tissue or limit the range of motion (ROM) (Tate [5]) resulting in joint dysfunctionality. Pathological changes in the knee joint can occur as a consequence of the ICR path change during the motion (Gerber and Matter [6]). For a joint rehabilitation device, whose purpose is restoring the proper ROM, function of muscles, nervous system, as well as supporting the regeneration of tissue, bones and cartilage by bringing them under naturally occurring loads, it is necessary to take into account the complex movement. Its omission could entail unforeseen negative consequences for the health of the patient or at least contribute to ineffective treatment. As a result, abnormal motion, inadequate ROM or joint motion pattern different from the intended and from the one occurring in a healthy patient's leg can persist. Harmful compensation mechanisms may be also developed, meaning exaggerated usage of for instance other muscles and joints to compensate for the lack in the knee joint's movability and ROM (Ogrodzka et al. [7]).

On the other hand, the possibility of considering the knee joint only approximately as a hinge is acknowledged in some cases in biomechanics (Huston [8]). Moreover, the amount of publications and research in this field like for example (Gerber and Matter [6]; Wiczkowski and Skiba [9]; Nägerl et al. [10]) proves that this difference in the motion sometimes is considered very little and is omitted, but it is something that should not be forgotten not only in prosthetic applications, but also when dealing with devices closely cooperating with the human knee joint during movement.

\section{Motivation and problem formulation}

In the case of the knee joint, there are solutions which can reproduce the joint's true motion in a significantly precise way. In terms of functionality, those devices can be categorized into two types. First, 
devices to some extent universal are designed for people meeting certain physical and anatomical requirements. One of them is Kincom that is a device using a polycentric hinge with a Chebyshev mechanism to approximately trace the ICR trajectory (Kim et al. [11]). Another one is Geo-Flex knee that is a friction-controlled polycentric knee. It was created for amputees who need stability and want to avoid a manual locking knee (Wiest [12]).

The other group is constituted by devices that in most cases are adapted to individual features of the patient joint's structure as for instance to the leg segments' lengths. Those devices include prostheses which must reflect accurately enough the patient's leg movements, so as not to cause discomfort, particularly with regard to the patient's other healthy leg's motion. Solutions of this type are often based on the so called "bionic-knee", which tries to precisely mimic the human natural movements by imitating the construction of a human joint. The shapes and structure of bones and even the meniscus operation are included ensuring an adequate flexion/extension movement compatible with the motion of the human knee (Nägerl et al. [13]). However, due to the fact that for each person this movement is characterized by the specific ICR centrode occurring throughout the change of knee's flexion angle, the device has to be adapted to individual features by differentiating its structure in geometry and shape. This way the knee joint used in devices supporting locomotion and kinesiotherapy can be built. Frequently, those devices are also based on solutions using gears (Lovasz et al. [14]; Gastaldi et al., [15]) or a cam mechanism with appropriately shaped elements (individual for each patient) enabling achievement of the user's natural flexion/extension characteristic.

Both groups of devices have some limitations and drawbacks. In the first type they do not enable full reproduction of the joint's movement and because of the application only to a certain group of people, the number of potential users becomes diminished. The other mentioned group attempts to overcome these disadvantages by manufacturing equipment for an individual patient, but this entails the need to produce another device for each new user or at best a fairly substantial reconstruction, which cannot be performed quickly by medical personnel. It also cannot be forgotten that along with the therapy's progress the patient's individual characteristics concerning the rehabilitated joint or the required rehabilitation movements may be altered. Thus, there is a need to readjust the device to the patient or rehabilitation with not exactly appropriate characteristics of a device will be performed. All these aspects contribute to limiting the possibility of application and the obtained rehabilitation results for both groups of devices.

For the above reasons, this paper is focused on the design of a novel mechanism for the knee joint having the capability of dynamic adaptation in real-time to the required ICR trajectory. The designed device will be able to step by step improve the motion adjusting little by little the trajectory to the correct one. The objective of the study consists in creating a device that can be a support for the knee joint's rehabilitation by finding an appropriate kinematic structure to check and show the feasibility of the designed solution. Simulations as well as evaluation of the device's operation and capabilities have been carried out.

\section{The mechanism's design}

\subsection{Topology search and design optimization}

Type synthesis was conducted aiming to find a novel mechanism solution for the knee joint by improving mimicking the movement that is performed by a human knee, particularly through the achieved ICR trajectory. The search has been performed with a formalized intermediate chain method to obtain the possible solutions, graphic forms of intermediate chains and basic schemes. At the beginning a simplified mechanism for the knee was searched with 1 DOF. The exploration process and the variety of achieved mechanisms is presented in (Olinski et al. [16]). A set of kinematic schemes of the mechanism (Fig.3) has been found and the solution B was chosen as the most appropriate and useful for adapting it to the complex knee movement introducing additional DOFs. This solution is the mentioned model of the knee joint that is based on a four-bar mechanism. The introduced improvement is based on variable length elements with prismatic joints instead of two bars $\left(L_{2}, L_{3}\right)$. This way solution Ba (Fig.4) has been obtained. The proposed new mechanism can be characterised as based on a modified Chebyshev mechanism with 2 additional DOFs having a total of 3 DOFs, to get a controlled adaptive polycentric hinge. 


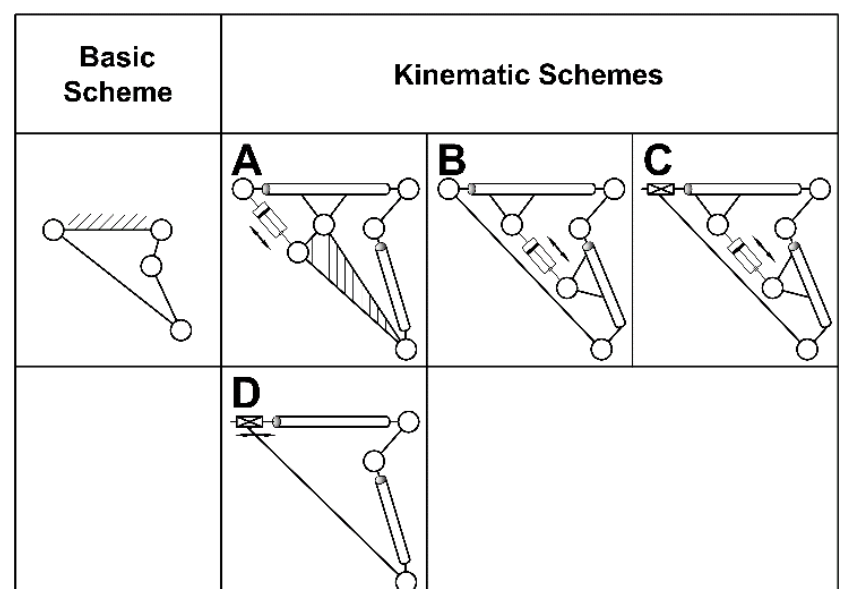

Fig.3. Examples of searched variety of the mechanisms (Olinski et al., 2015).

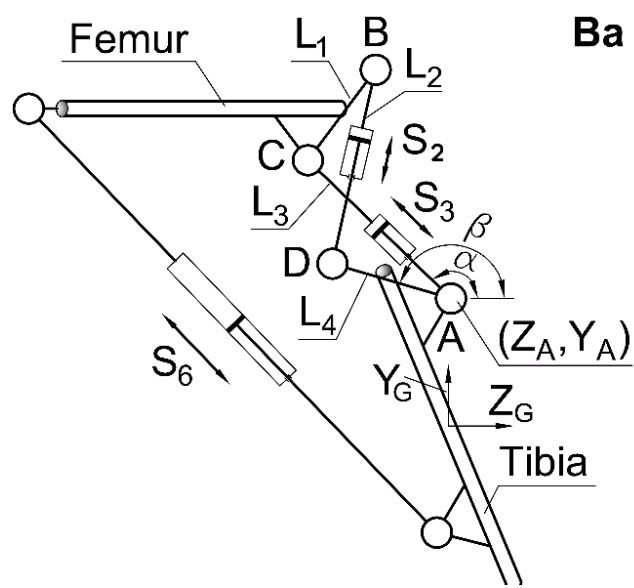

Fig.4. Kinematic scheme of the original proposed mechanism with translational motors.

Furthermore, another possibility of introducing additional DOFs has been studied. A mechanism with the variable length element has been worked out as a two-bar mechanism with rotational joints in the form of solution $\mathrm{Bb}$ (Fig.5) with nearly the same design parameters, but with rotational motors $R$ instead of cylinders $S$. Further research and simulations were performed with the original solution, since its structure was considered less complicated and more suitable for accurate control of the movements. However, the second solution will be investigated in future research, since it may occur to be easier to apply and may offer a wider range of the reachable ICR trajectories as due to the limited cylinders' strokes.

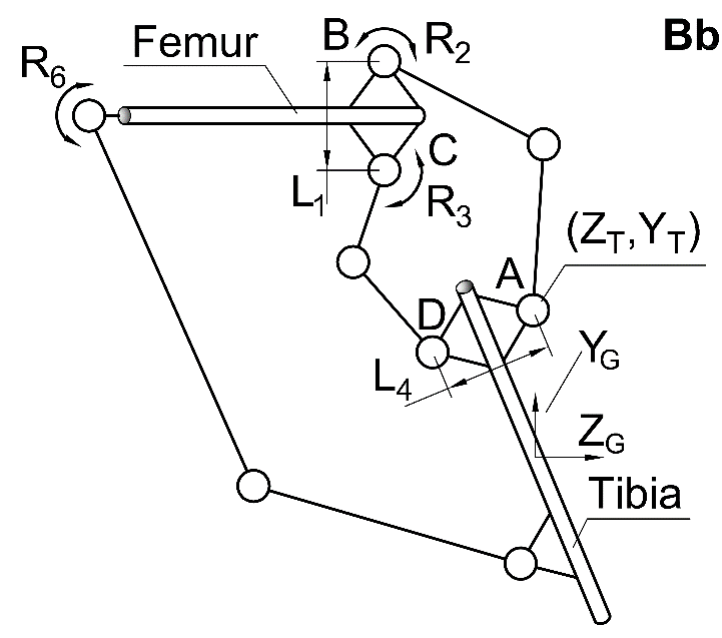

Fig.5. Kinematic scheme of the modified proposed mechanism with rotational motors.

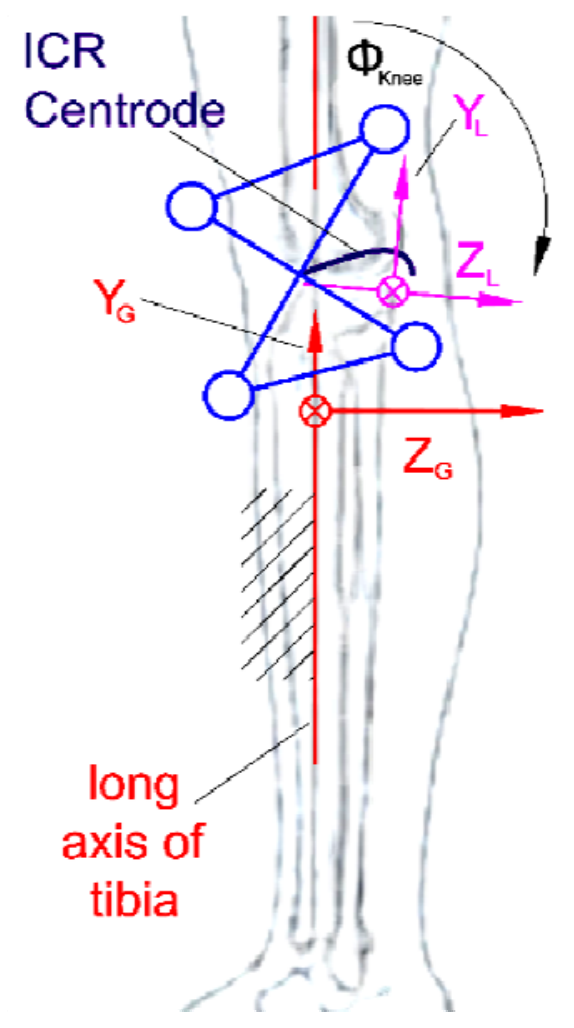

Fig.6. The knee's coordinate systems used for specifying the reference ICR trajectories. 
The idea of introducing variable length elements in a crossed four-bar mechanism for a better knee motion reproduction is not completely new. For instance, it has been mentioned in (Moser [17]), but it was not developed further, since it was considered too complicated and possibly shaky. However, the mentioned potential problems are considered in this paper by reducing the number of variable length elements to only 2 crossed bars and introducing full control of their lengths by applying mechatronic motions (translational or rotational).

As a next step an optimal kinematic synthesis of the mechanism dimensions has been performed by specifying the design of the structure, especially the dimensions of a four-bar linkage capable of reproducing the ICR trajectory close to the actual displacement in the human knee. The equations 3-8 in the article (Walker [18]) describe the average displacement of ICR according to the flexion of the knee joint in the range of $0-120^{\circ}$. These relations were used in article (Bertomeu et al. [19]) to generate the trajectory of the knee's ICR in the sagittal plane $(y, z)$ in a computed plot. This particular curve was adapted as a reference ICR trajectory called Walter et al. model (Bertomeu et al. [19]). In this paper, the trajectory had to be properly modified to fit the point of origin and orientation of the designed structure's new coordinate system $\{\mathrm{G}\}$ (Fig.6). The used local coordinate system $\{\mathrm{L}\}$ is the one in article (Bertomeu et al. [19]) with the $Z_{L}$ axis fixed with the tibia bone's surface, whereas the global system $\{\mathrm{G}\}$ is fixed with the leg's axes of the sagittal plane $Y_{G}, Z_{G}\left(Z_{G}\right.$ coincides with the tibia bone's long axis). In order to achieve the correct shape of the reference curve it was rotated by $10^{\circ}$ and translated along $y, z$ axes. As a result, the obtained reference ICR trajectory was used during the optimization process and later for simulations. It is presented in the plot (Fig.7 - I) together with other 2 close example trajectories (II, III), since the ICR trajectory is specific for each person. In addition, the two placed broader boundary trajectories (IV, V) are presented and applied during simulations as a reference for evaluating the capabilities of the designed mechanism in generating different shapes and ranges of trajectories.

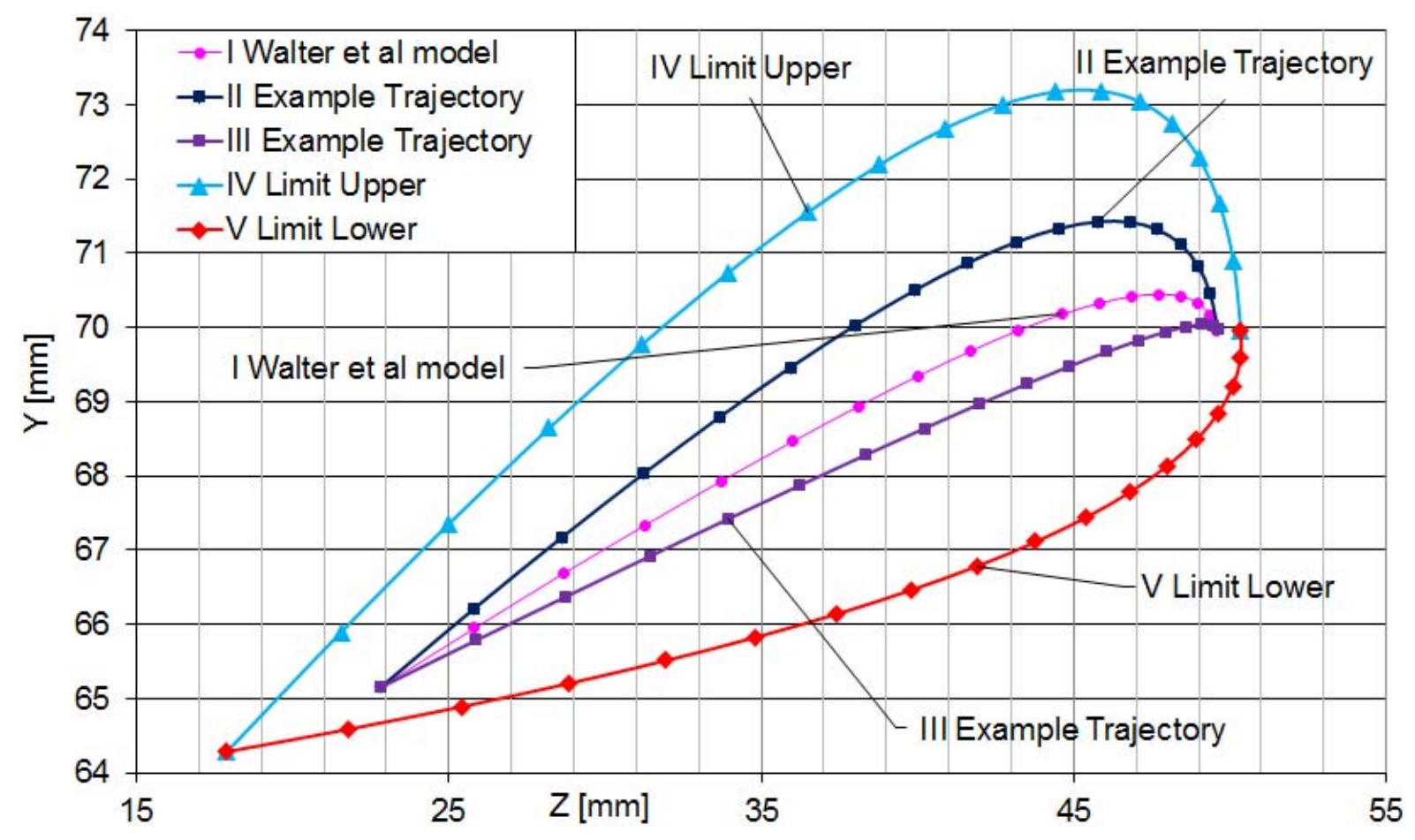

Fig.7. The ICR trajectories used as reference during simulations.

In order to achieve the structure capable of generating the chosen reference ICR trajectory the mechanism elements' dimensions of the four-bar mechanism were optimized. The optimization of the fourbar linkage design for a specific path was described in (Buśkiewicz [20]) by means of evolutionary 
algorithm. However, in this paper an optimization was started using the SAM ARTAS - Engineering Software with the initial dimensions of the crossed four-bar mechanism from the device Kincom (Kim et al. [11]). The motion was imposed on the femur in the range of 0 to $120^{\circ}$ with an appropriate equivalent mechanism, whereas the used sliders, connected by a revolute joint, constituted equivalent mechanism for determining the ICR position (Fig.8). The mentioned curve based on Walter et al model (Fig.7 - I) was used as a reference trajectory. By changing the positions of the elements' vertexes in SAM a mechanism performing approximately the desired motion and nearly realizing the reference ICR trajectory was reached as reported in Fig.8.
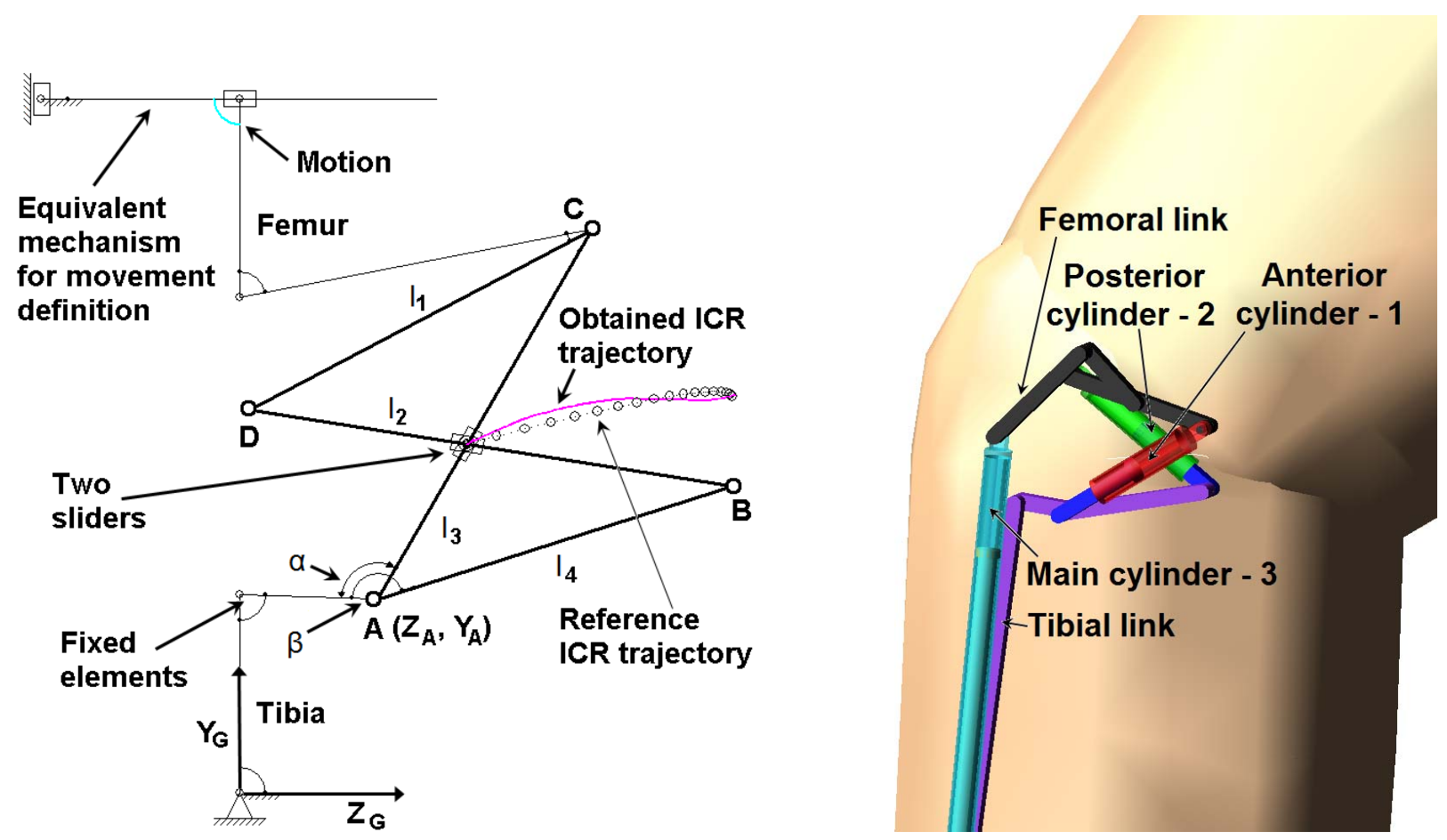

Fig. 8. The approximately optimal model built in SAM software with acquired (solid line) and reference (dotted line) ICR trajectories.

Fig.9. The final mechanism's model in Adams with optimal dimensions and variable length crossed bars.

The computed dimensions were used as a starting point for building a crossed four-bar numerical model in Adams software and for performing further thorough optimisation. The aim of optimisation was to decrease the difference between the obtained and reference ICR trajectories. This requirement was formed by formulating the objective function

$$
f_{M I N}=\sum_{i=1}^{n}\left(\sqrt{\left(Z_{R}-\mathrm{Z}_{\mathrm{O}}\right)^{2}+\left(Y_{R}-\mathrm{Y}_{\mathrm{O}}\right)^{2}}\right)_{i}
$$

where $Z_{R}, Y_{R}, Z_{O}, Y_{O}$ are the $(Z, Y)$ coordinates of reference and obtained trajectory's subsequent points. The optimised variables included $l_{1}, l_{2}, l_{3}, l_{4}, Z_{A}, Y_{A}, \alpha$ and $\beta$ (Fig.8). During the optimisation process the values of variables were changed in search of the objective function's minimum average value. After obtaining the optimal dimensions enabling a fairly accurate generation of the reference trajectory, the numerical model in Adams was modified, according to the previously chosen kinematic scheme (Fig.4), by adding 2 DOFs in the form of variable length crossed bars to get the final design of the mechanism shown in Fig.9. This model was applied for performing couple of simulations in order to evaluate its movement's accuracy and capabilities. 


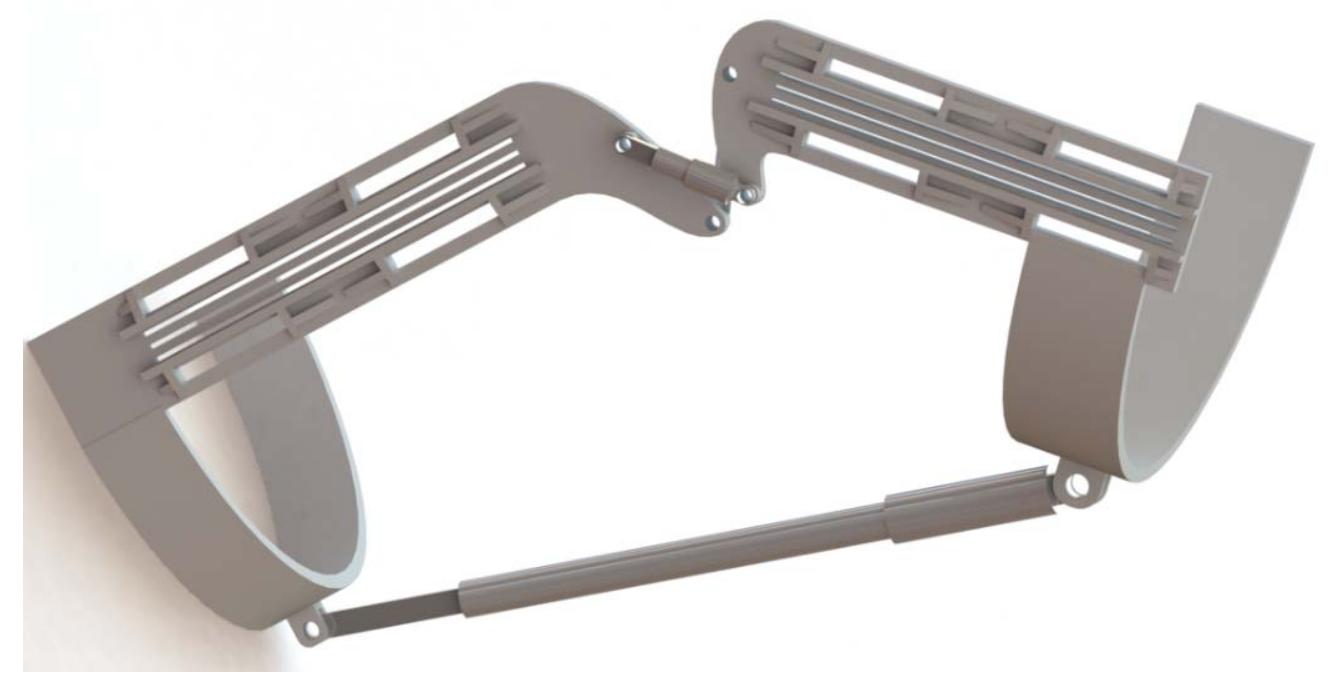

Fig.10. The CAD shape design of the mechanism applied to a knee joint rehabilitation supporting device modelled in Solidworks.

The mechanical interface was also computed. Basing on the obtained adaptive model a CAD design and assembly of novel device for the knee joint was elaborated in the Solidworks program as shown in Fig. 10 in order to design the elements' shapes more precisely.

\subsection{Basic performance of designed mechanism}

The novel designed mechanism will have the possibility of a very accurate adjustment of the ICR trajectory to individual needs thanks to the additional DOFs. The obtained ICR trajectory is estimated to be characterized by a very small deviation from the desired natural movement for the human knee. The possibility of real-time adjustment of the mechanism's elements (lengths of crossing bars) to control the position of the ICR can be considered one of the main advantages. Thus, an improvement of the rehabilitation process can also be predicted by increasing the patient's comfort and reducing the level of pain, since the proper trajectory of knee joint's ICR movement can be achieved in little steps. As for finding the mentioned proper trajectory the biomechanics of the patient's second knee joint can be treated as a reference and this could be measured by the same device. In many cases, the proper reference trajectory may be also provided by a qualified physiotherapist, who will guide the leg in an appropriate manner during a reference training session. This movement can be then measured, saved and reproduced by the device.

The mechanism will be especially useful when dealing with various kinds of soft injuries like twists or ligaments and tendons' injuries, e.g., damage to collateral ligament (Tate [5]). The reason is the fact that in such cases the biomechanics of the joint can be distorted and the natural movement of the knee may not occur. The designed novel mechanism is expected to be applied in various devices for the human knee support (orthoses) and rehabilitation devices, possibly even as knee replacements (implants) to adjust in real time to the proper knee motion.

\section{Results of simulations}

The final model of the designed adaptive crossed four-bar mechanism in Fig.9 was used for performing dynamic simulations in Adams. The numerical model was loaded vertically on the femoral link with $V=1 \mathrm{kN}$ force simulating the human body weight. The degree of compatibility between the obtained and the reference ICR trajectories was studied particularly in terms of the trajectories deviation. Moreover, the cylinder's strokes and force ratios were determined. 
Table 1. Numerical results of simulations.

\begin{tabular}{|c|c|c|c|c|c|c|c|}
\hline \multirow{2}{*}{ No. } & $\begin{array}{c}\text { The model / } \\
\text { Reference } \\
\text { trajectory (Fig.7) }\end{array}$ & \multicolumn{3}{|c|}{ Cylinder's stroke } & \multicolumn{3}{c|}{ Cylinder's force ratio } \\
\cline { 3 - 8 }$[\mathrm{mm}]$ & $\begin{array}{c}\Delta S_{2} \\
{[\mathrm{~mm}]}\end{array}$ & $\begin{array}{c}\Delta S_{3} \\
{[\mathrm{~mm}]}\end{array}$ & $\begin{array}{c}F_{1} / V \\
{[-]}\end{array}$ & $\begin{array}{c}F_{2} / V \\
{[-]}\end{array}$ & $\begin{array}{c}F_{3} / V \\
{[-]}\end{array}$ \\
\hline 1. & $\begin{array}{c}4 \text { bar model / } \\
\text { I Walter et al }\end{array}$ & - & - & 78.06 & - & - & 0.70 \\
\hline 2. & $\begin{array}{c}\text { Adaptive model / } \\
\text { I Walter et al }\end{array}$ & 11.02 & 5.64 & 78.06 & 0.93 & 1.59 & 0.76 \\
\hline 3. & $\begin{array}{c}\text { Adaptive model / } \\
\text { IV Limit Upper }\end{array}$ & 20.02 & 10.38 & 81.74 & 1.05 & 0.19 & 0.92 \\
\hline 4. & $\begin{array}{c}\text { Adaptive model / } \\
\text { V Limit Lower }\end{array}$ & 21.77 & 15.91 & 81.70 & 1.10 & 1.92 & 1.04 \\
\hline
\end{tabular}

Four experiments are listed in Tab.1, whose results were illustrated with appropriate plots and derived numerical values. First experiment was conducted with the four-bar mechanism with optimal dimensions, but still without the variable length elements. The results obtained in this case (Fig.11) showed that the reference trajectory could be approximately achieved and the maximum relative error in $z, y$ axes was equal to $7.51 \%$ and $0.97 \%$ respectively.

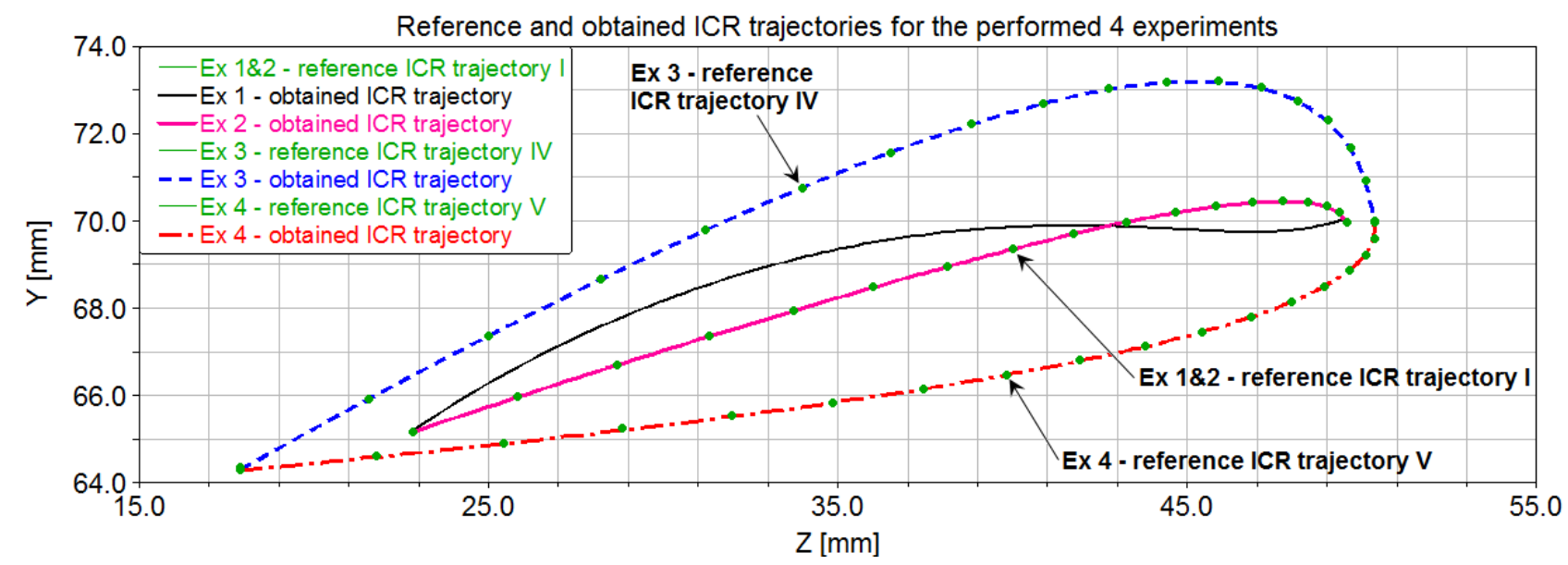

Fig.11. Comparison of the reference ICR trajectories (green marks indicate their points) with the obtained trajectories from 4 simulations in Adams.

Further three experiments were conducted on the final adaptive mechanism in reference to the chosen trajectories (Fig.7 - I, IV, V). In these simulations it was observed that the desired trajectory could be exactly achieved (Fig.11). The upper and lower limit trajectories (IV, V) were achieved during experiments number 3 and 4 (Fig.11). The results suggest that nearly any trajectory within the area formed by two boundary trajectories can be achieved using the developed mechanism. This statement is also further supported by the precise numerical results, derived from (Fig.11), showing very little deviation from the reference trajectories. 


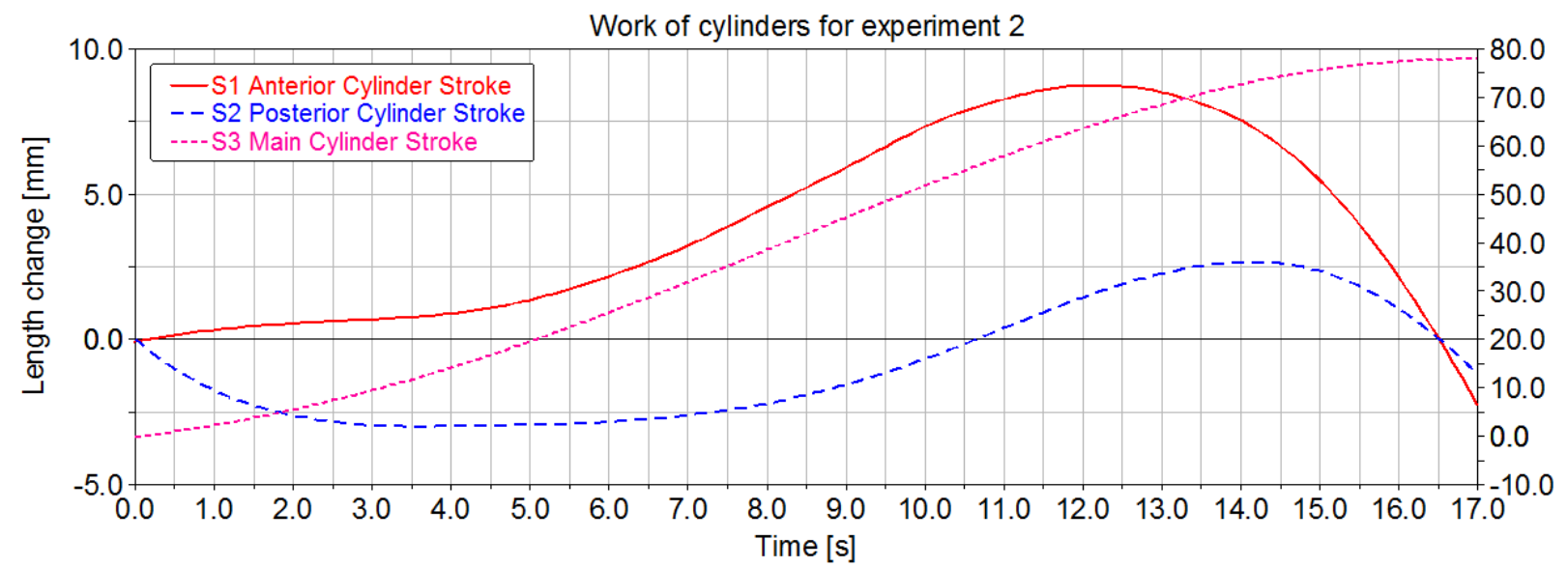

Fig.12. Experiment No. 2 - the length changes of the cylinders.

The conducted simulations were used also for measuring the length changes in the mechanism cylinders (Fig.12 - Fig.13) and force ratios exerted in these motors. As an example force ratios for the second experiment were presented in plot (Fig.14).

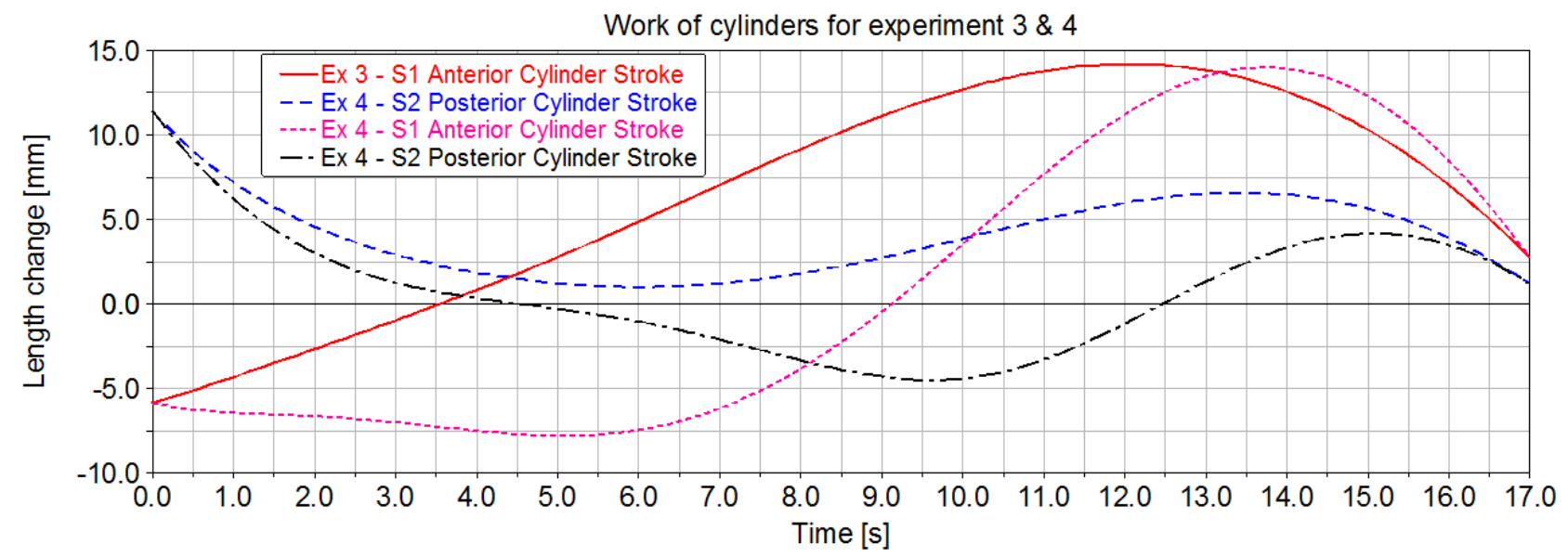

Fig.13. Experiment No. 3 and No. 4 - the length changes of the anterior and posterior cylinders.

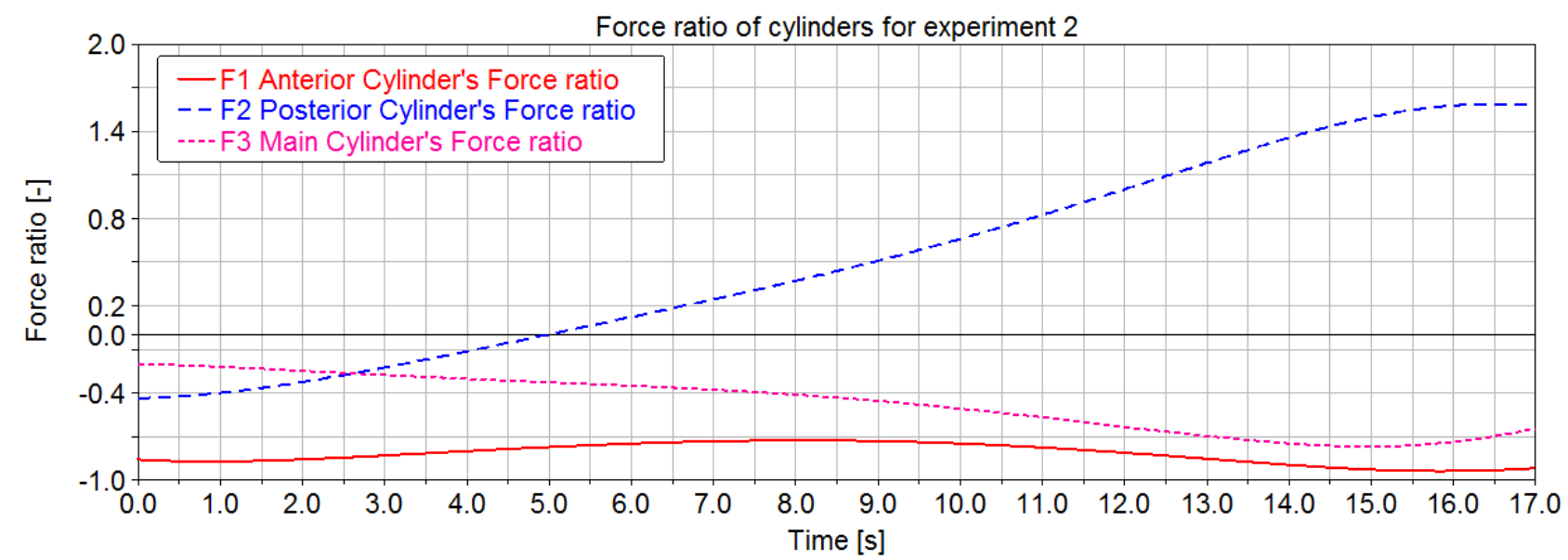

Fig.14. Experiment No. 2 - the measured force ratios exerted by cylinders. 
The maximum strokes of the anterior, posterior and main cylinders were obtained during all simulations and they were approximately equal to $21.77 \mathrm{~mm}, 15.91 \mathrm{~mm}$ and $81.74 \mathrm{~mm}$ respectively, whereas, the maximum absolute value of the force ratio required by cylinders was computed as 1.10, 1.92 and 1.04 respectively. The results of cylinder strokes indicate that it is possible to build the designed mechanism with variable length bars, since they are sufficiently small to fit in the limited space. However, in order to achieve an appropriate resolution of control a special type of motor might be required.

\section{Discussion}

The designed mechanism renders it possible to control the movement of the ICR and to generate accurately various desired reference trajectories. The proposed mechanism constitutes a compromise solution between the complexity of the mechanism and the accuracy of the human anatomical movement reproduction. On the one hand, it is a simplified model of the human knee mechanism concentrating on the main flexion/extension movement just in the sagittal plane. In this way it constitutes a simplification of already developed complicated and complex models taking into consideration the 3D movement, meniscus, patella (Ciszkiewicz and Knapczyk [21]) and other elements that are based mainly on bone surface cooperation (Ciszkiewicz and Knapczyk [2]). On the other hand, the proposed solution is an advance with respect to the other existing mechanisms, since it takes into consideration the complexity (roll and slide) of flexion/extension movement.

The advantage of this mechanism over those already existing is mainly the structure that is simplified to planar motion, but nonetheless it has the ability to reproduce the human knee movement with a satisfying accuracy. Above all, it can be controlled and it allows real-time trajectory's modification and adjustment, since the mechanism's geometry can be changed accordingly to the needs during user's motion in order to achieve movement of a better quality. Even many existing mechanisms that reproduce the movement in 3D space lack this possibility, which may be very useful while restoring the proper human movement. In many cases this kind of simplified mechanism can produce a sufficiently accurate pattern of motion.

The question whether the application of just one additional DOF instead of 2 DOFs could be sufficient for achieving the desired range of trajectories is still open. There is a possibility that if just one of the crossed bars is length-variable, then the obtained trajectories could be less accurate and also the range of capabilities could be limited. It would cause a greater stroke and force demand from the one introduced cylinder. For this reason, for initial research it was chosen to use 2 additional DOFs to be able to achieve a wide range of trajectories.

Regarding its application, such mechanism, exactly imitating the movement of the knee's rotation axis during flexion, may be suitable for correcting motion pattern and the ICR trajectory for soft injuries associated with muscles, ligaments, etc. As regards the hard injuries related with fractures, which may have not been completely properly set, there are rather no attempts to intervene in the occurring incorrect motion axis by exercises alone.

The adaptation of the mechanism for many people with different ranges of the ICR trajectories is also possible. However, this range is limited, since the required trajectories may reach beyond the achievable space. The solution to this issue could be a modular structure of the mechanism with interchangeable elements of different lengths. This way by replacing some of the mechanism's elements, the range of achievable trajectories could be expanded and the mechanism could be adapted to more individual needs. Nevertheless, the main advantage of the proposed mechanism is the possibility of real-time control and obtaining accurate trajectory that may be changed little by little.

The specific device presented in this article, using the novel designed mechanism can be considered at first to be used for the kinesiotherapy of the knee joint and once further developed for human movement measurements. Moreover, the designed mechanism is not only useful in rehabilitation and understanding human biomechanics, but also is likely to find application in prostheses, implants, as well as robotics, especially humanoid robots and exoskeletons. 


\section{Conclusions}

Anatomy and biomechanics of the human knee joint have been studied to identify and characterize the complex movement in the human knee as a combination of roll and slide. Requirements and problems for knee rehabilitation assisting device have been determined. These include, among others, generating physiological movement with particular attention to the ICR displacement with the joint's full ROM, since the application of mechanisms incompatible with human joints may damage the internal tissue, limit the joint's ROM, as well as cause pain and discomfort to the user.

The work on designing a novel device for the human knee joint included expanding the already completed part of type synthesis. In order to reproduce the ICR trajectory two possible novel solutions, based on linear and rotational actuators, were obtained. The mechanism's dimensions were optimised to minimise the difference between the reference and obtained ICR trajectories. An adaptive model of crossed four-bar mechanism has been designed, its numerical model was built in Adams and the shape design has been performed by making a preliminary model in Solidworks. Kinematics of the novel mechanism provide good reproduction of the physiological movement. One of the main objectives, i.e., accomplishing the given motion with very minor deviation was achieved, since a very small trajectory deviation of the knee's ICR from the reference trajectory has been computed during simulations. Furthermore, the simulation results of cylinders required strokes and forces suggest that it should be possible to build such a device. Above all, the possibility of adjustment in real-time to individual patients' needs is provided by the mechanism. The expected improvements in rehabilitation process include the possibility of restoring the physiological movement, increasing comfort and reducing pain by correcting the trajectory in little steps.

Simulations were already worked out, but further research and work in this field will be necessary. The final research step would be building a prototype and testing the mechanism's capabilities.

\section{Aknowledgements}

Part of this work has been developed during an internship of the first author at LARM in Cassino, Italy, in 2015, with a scholarship within the project NJK at the Faculty of Mech. Eng. of Wrockaw University of Science and Technology supported by the EU. The first author wishes to thank the staff and students at LARM for their support.

\section{Nomenclature}

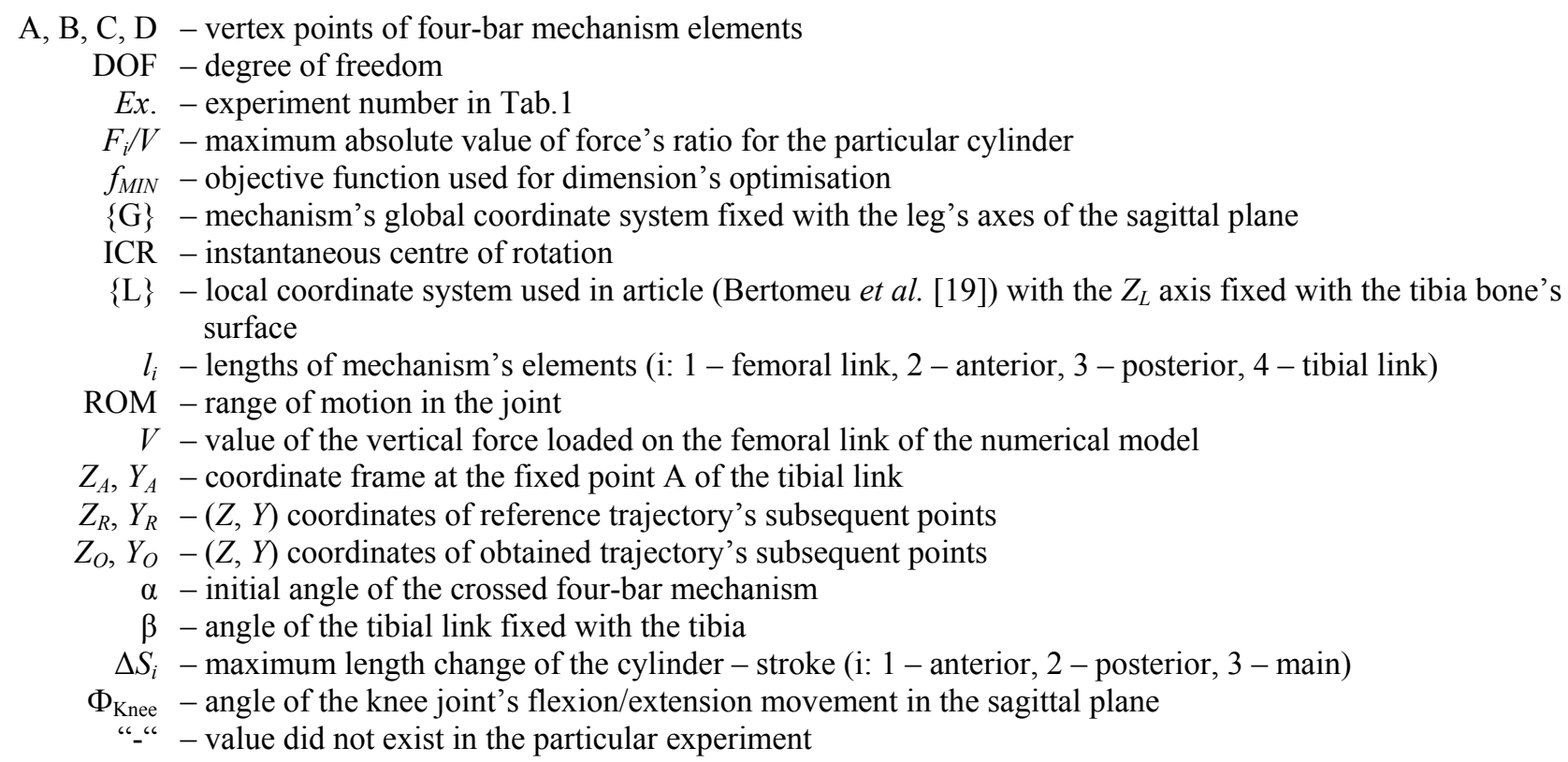




\section{References}

[1] Varela M., Ceccarelli M. and Flores P. (2015): A kinematic characterization of human walking by using CaTraSys. Mechanism and Machine Theory, vol.86, pp.125-139.

[2] Ciszkiewicz A. and Knapczyk J. (2014): Parameters estimation for the spherical model of the human knee joint using vector method. - Int. J. of Applied Mechanics and Engineering, vol.19, No.3, pp.523-537. DOI: 10.2478/ijame-2014-0035.

[3] Lovasz E.C., Pop C., Pop F. and Dolga V. (2014): Novel solution for leg motion with 5-link belt mechanism. - Int. J. of Applied Mechanics and Engineering, vol.19, No.4, pp.699-708. DOI: 10.2478/ijame-2014-0048.

[4] Liang C., Ceccarelli M. and Takeda Y. (2012): Operation analysis of a Chebyshev-Pantograph leg mechanism for a single DOF biped robot. - Front. Mech. Eng. vol.7, No.4, pp.357-370. DOI 10.1007/s11465-012-0340-5.

[5] Tate P. (2012): Seeley's Principles of Anatomy \& Physiology. - Second Edition. ISBN: 0073378194.

[6] Gerber C. and Matter P. (1983): Biomechanical analysis of the knee after rupture of the cruciate ligament and its primary repair. An instant-centre analysis of function. - The J. of Bone and Joint Surgery, vol.65-B, No.4, pp.391-399.

[7] Ogrodzka K., Niedźwiedzki T. and Chwała W. (2011): Evaluation of the kinematic parameters of normal-paced gait in subjects with gonarthrosis and the influence of gonarthrosis on the function of the ankle joint and hip joint. Acta of Bioengineering and Biomechanics, vol.13, No.3, pp.47-54.

[8] Huston R.L. (2008): Principles of Biomechanics. - CRC Press, ISBN: 978-0-8493-3494-8.

[9] Wiczkowski E. and Skiba K. (2008): Kinetic analysis of the human knee joint. - Biology of Sport, vol.25, No.1, pp.77-91.

[10] Nägerl H., Dathe H., Fiedler CH., Gowers L., Kirsch S., Kubein-Messenburg D., Dumont C. and Wachowski M.M. (2015): The morphology of the articular surfaces of biological knee joints provides essential guidance for the construction of functional knee endoprostheses. - Acta of Bioengineering and Biomechanics, vol.17, No.2, pp.45-53.

[11] Kim K.J., Kang M.S., Choi Y., Jang H.Y., Han J. and Han C. (2012): Development of the exoskeleton knee rehabilitation robot using the linear actuator. - International Journal of Precision Engineering and Manufacturing, vol.13, No.10, pp.1889-1895, DOI: 10.1007/s12541-012-0248-3.

[12] Wiest J. (2002): What's new in Prosthetic Knees? - In Motion, vol.12, No.3.

[13] Nägerl H., Frosch K.H., Wachowski M.M., Dumont C., Abicht CH., Adam P. and Kubein-Meesenburg D. (2008): A novel total knee replacement by rolling articulating surfaces. In vivo functional measurements and tests. Acta of Bioengineering and Biomechanics, vol.10, No.1, pp.55-60.

[14] Lovasz E.C., Modler K.H., Draghici A. and Vacarescu V. (2009): Studies for a new prosthesis design for the work capacity rehabilitation. - Annals Of DAAAM and Proceedings, pp.1549-1550.

[15] Gastaldi L., Lisco G. and Pastorelli S. (2015): Evaluation of functional methods for human movement modelling. Acta of Bioengineering and Biomechanics, vol.17, No.4, pp.31-38.

[16] Olinski M., Lewandowski B. and Gronowicz A. (2015): Type synthesis and preliminary design of devices supporting lower limb's rehabilitation. - Acta of Bioengineering and Biomechanics, vol.17, No.1, pp.117-127.

[17] Moser S. (2013): Development of a Variable Knee Joint. - Bachelor-Thesis, ETH, Zurich.

[18] Walker P.S., Kurosawa H., Rovick J.S. and Zimmerman R.A. (1985): External knee joint design based on normal motion. - J. Rehabil. Res. Dev., vol.22, No.1, pp.9-22.

[19] Bertomeu J.M.B., Lois J.M.B., Guillem R.B., Pozo A.P.D., Lacuesta J., Mollà C.G., Luna P.V. and Pastor J.P. (2007): Development of a hinge compatible with the kinematics of the knee joint. - Prosthetics and Orthotics International, vol.31, No.4, pp.371 - 383. http://dx.doi.org/10.1080/03093640601095842.

[20] Buśkiewicz J. (2014): A specific problem of mechanism synthesis. - Int. J. of Applied Mechanics and Engineering, vol.19, No.3, pp.513-522. DOI: 10.2478/ijame-2014-0034.

[21] Ciszkiewicz A. and Knapczyk J. (2015): Parameters Estimation for a patellofemoral joint of a human knee using a vector method. - Int. J. of Applied Mechanics and Engineering, vol.20, No.3, pp.629-636.

Received: June 19, 2016

Revised: July 3, 2016 